\title{
Cell-Free Massive MIM0: Distributed Signal Processing and Energy Efficiency
}

Zakir Hussain Shaik

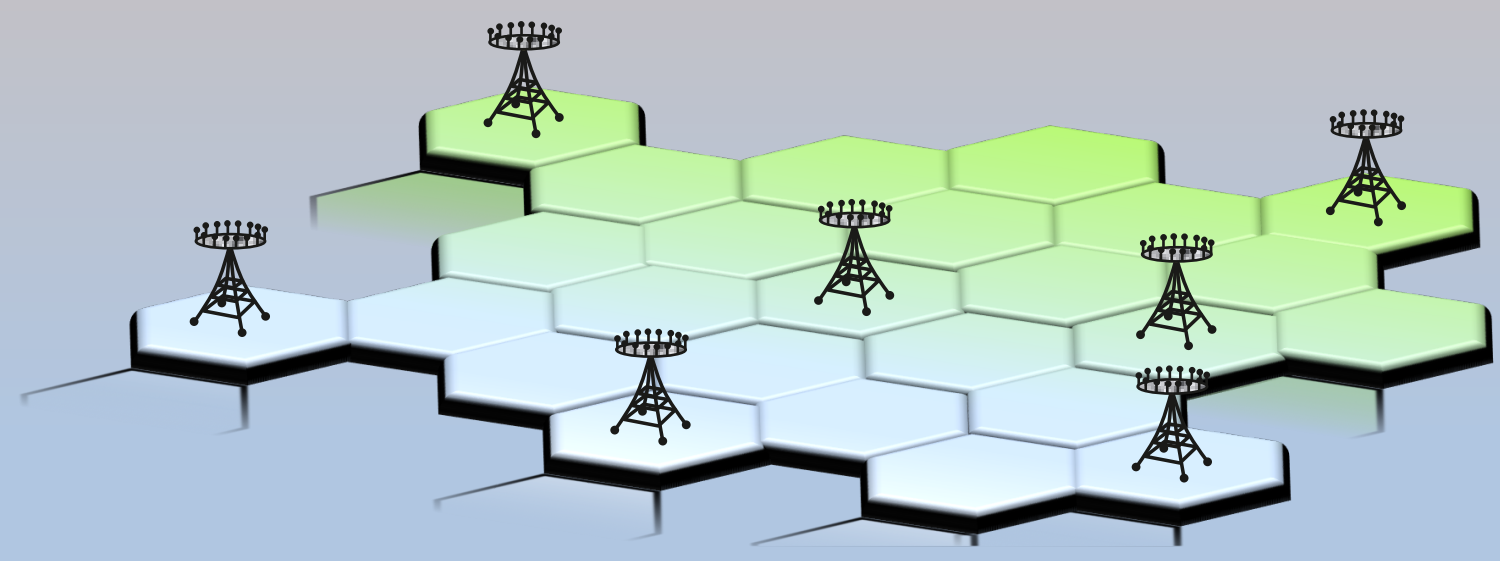





\title{
Cell-Free Massive MIMO: \\ Distributed Signal Processing and Energy Efficiency
}

\author{
Zakir Hussain Shaik
}

\section{COMMUNiCATION SŶstems}

Division of Communication Systems Department of Electrical Engineering (ISY) Linköping University, 58183 Linköping, Sweden www.commsys.isy.liu.se

Linköping 2022 
This is a Swedish Licentiate Thesis.

The Licentiate degree comprises 120 ECTS credits of postgraduate studies.

Cover: A large geographical area depicting cell-free network wherein multiple access points with multiple antennas (possibly Massive MIMO) serve all the users in the area instead of having a single access point in each cell as in a cellular network. Darker boundary indicates cluster border, and transparent cell edges highlight that we move beyond cellular network architectures and allow for all the access points to jointly and coherently operate as a cell-free network.

(c) EY-No This work is licensed under a Creative Commons AttributionNonCommercial 4.0 International License.

https://creativecommons.org/licenses/by-nc/4.0/

Cell-Free Massive MIMO: Distributed Signal Processing and Energy Efficiency

(c) 2022 Zakir Hussain Shaik, unless otherwise stated.

ISBN 978-91-7929-222-5(print)

ISBN 978-91-7929-223-2(PDF)

ISSN 0280-7971

URL https://doi.org/10.3384/9789179292232

Printed in Sweden by LiU-Tryck, Linköping 2022 


\section{Abstract}

In this era of rapid wireless technological advancements, wireless connectivity between humans, humans with machines, and machines with machines is gradually becoming an absolute necessity. The initial motivation for wireless connectivity was to enable voice communication between humans over a geographical area. Thanks to cellular communications advancements in the past decade, cellular wireless connectivity has become a global success, starting from $1 \mathrm{G}$ to the present generation 5G. However, the needs of humans often evolve with time, and now the world is witnessing an ever-growing demand for the internet with high data rates besides reliable voice communication. Current cellular networks suffer from non-uniform data rates across a cell, i.e., users at the cell center and the cell edges experience significant variations in signal-to-noise ratio, making the cellular technology less reliable to meet the future data demands. Moreover, cellular networks operating as cells, i.e., an access point (AP, the term we would use instead of base station) serving the users within its geographical location, cannot leverage the network's total capacity without cooperation among APs of the neighboring cells. One potential solution is moving away from the cell to cell-free networks wherein all the APs will serve all the users within the geographical coverage area. Thus, there is a need for a paradigm shift in how cellular networks operate. Towards the goal mentioned above to fully leverage the network capacity, the Cell-Free Massive multiple-input-multiple-output (MIMO) technology is expected to be the next potential technology beyond 5G combining the benefits of Massive MIMO and cell-free distributed architectures.

Distributed architectures require distributed signal processing algorithms, and also energy consumption of the network is crucial. Keeping in view the practical ease in deployment, we consider a sequentially connected CellFree Massive MIMO network called a "radio stripe". In the first part of the thesis, we focus on developing an optimal sequential algorithm in the sense of mean-square-error (MSE) which has the same performance as that of centralized Cell-Free Massive MIMO implementation with the 
minimum MSE (MMSE) receiver. We also develop an optimal sequential algorithm that decentralizes the centralized bit LLR computation. Another attractive aspect of these proposed algorithms is that the fronthaul (number of real symbols required by the central processing unit (CPU) to decode the transmitted signal) is independent of the number of APs. On the contrary, centralized implementation fronthaul is dependent on the number of APs, causing scalability problems with the increase in APs.

In the second part of the thesis, we develop an algorithm focused on maximizing the energy efficiency of the RadioWeave network in an underlay spectrum sharing. RadioWeave is a technology envisioned to combine CellFree Massive MIMO and possibly large intelligent surfaces. We first present the energy efficiency problem, which is non-convex in its original form. Then, a convex lower bound on the problem is provided with an iterative algorithm to solve the problem efficiently. 


\section{Populärvetenskaplig Sammanfattning}

I denna tid av snabba trådlösa tekniska framsteg blir trådlös anslutning mellan människor, mellan människor och maskiner och mellan maskiner och maskiner gradvis en absolut nödvändighet. Den ursprungliga motivationen för trådlös anslutning var att möjliggöra röstkommunikation mellan människor över ett stort geografiskt område. Tack vare framsteg inom mobilkommunikation under det senaste decenniet har mobil trådlös anslutning blivit en global framgång, från $1 \mathrm{G}$ till den nuvarande generationen 5G. Men människors behov utvecklats med tiden, och nu bevittnar världen, förutom pålitlig röstkommunikation, en ständigt växande efterfrågan på internet med höga datahastigheter. Nuvarande cellulära nätverk lider av olikformiga datahastigheter över cellen, d.v.s. användare i cellcentret och cellkanterna upplever betydande variationer i signal-brusförhållande, vilket gör den cellulära tekniken mindre lämplig för att möta framtida databehov. Dessutom kan cellulära nätverk som fungerar som celler, d.v.s. att varje accesspunkt (AP, termen vi använder istället för basstation) betjänar användarna inom dess geografiska område, inte utnyttja nätverkets totala kapacitet utan samarbete mellan AP:er i de angränsande cellerna. En potentiell lösning är att gå från cellulära till cellfria nätverk där alla basstationer betjänar alla användare inom det geografiska täckningsområdet. Det finns alltså ett behov av ett paradigmskifte i hur cellulära nätverk fungerar. För att fullt ut utnyttja nätverkskapaciteten, förväntas Cell-Free Massive MIMO vara nästa potentiella teknik bortom 5G som kombinerar fördelarna med Massive MIMO och cellfria distribuerade arkitekturer.

Distribuerade arkitekturer kräver distribuerade signalbehandlingsalgoritmer, och även energiförbrukningen i nätverket är av stor vikt. Vi studerar sekventiellt anslutna Cell-Free Massive MIMO-nätverk som kallas "radio stripe", eftersom dessa är enkla att placera ut. 
I den första delen av avhandlingen fokuserar vi på att utveckla en optimal, ur ett MSE perspektiv, sekventiell algoritm som har samma prestanda som den för centraliserad Cell-Free Massive MIMO-implementering med en MMSE-mottagare. Vi utvecklade också en optimal sekventiell algoritm som decentraliserar den centraliserade bit LLR. En kritisk aspekt av dessa föreslagna algoritmer är att fronthaul (antal reella symboler som krävs av CPU:n för att avkoda den sända signalen) är oberoende av antalet AP:er. Tvärtom är fronthaulen i en centraliserad implementering beroende av antalet AP:er, vilket orsakar skalbarhetsproblem med ökningen av AP:er.

I den andra delen av avhandlingen utvecklar vi en algoritm fokuserad på att maximera energieffektiviteten i RadioWeave-nätverk med en underliggande spektrumdelning. RadioWeave är en teknik som är tänkt att kombinera Cell-Free Massive MIMO och möjligen stora intelligenta ytor. Vi presenterar först energieffektivitetsproblemet, som är icke-konvext i sin ursprungliga form. Sedan förses en konvex nedre gräns för problemet med en iterativ algoritm för att lösa problemet effektivt. 


\section{Contents}

Acknowledgements $\quad$ ix

List of Abbreviations $\quad$ xi

1 Introduction 1

1.1 Background and Motivation ................. 1

1.2 Cell-Free Massive MIMO: A Blend of Best Existing Techniques 2

1.2.1 Benefits of Cell-Free Massive MIMO . . . . . . . . . . 4

1.2.2 Topology of Cell-Free Massive MIMO . . . . . . . . . 4

1.3 Thesis Outline .................. 5

2 Basics and Preliminaries $\quad 7$

2.1 System Model . . . . . . . . . . . . . . . . . . 7

2.1.1 Coherence Block and Block Fading . . . . . . . . 8

2.1.2 Time-Division Duplexing Protocol ......... . 9

2.1.3 Channel Model . . . . . . . . . . . . . . . . 10

2.1.4 Capacity Bounds as Performance Benchmark . . . . . 10

2.2 Cell-Free Massive MIMO: Channel Model and Features . . . 12

2.2.1 Spatially Correlated Channel Model . . . . . . . . . . 12

2.2.2 Channel Hardening and Favorable Propagation . . . . 12

2.3 Energy Efficiency .................. 16

2.3.1 Motivation and Definition . . . . . . . . . . . 16

2.3.2 Dinkelbach's Algorithm ............ . 17

3 Contributions of the Thesis $\quad 19$

3.1 Included Papers . . . . . . . . . . . . . . . . . . . 19

3.2 Excluded Papers .................... . 21

4 Conclusion and Future Scope $\quad 23$ 
Included Papers $\quad 32$

A MMSE-Optimal Sequential Processing 33

1 Introduction . . . . . . . . . . . . . . . 35

1.1 Related Work . . . . . . . . . . . . . . . 37

1.2 Contribution . . . . . . . . . . . . . 37

1.3 Paper Outline . . . . . . . . . . . . . . . . . 38

1.4 Notations . . . . . . . . . . . . . . . . . . 39

2 System Model And Channel Estimation . . . . . . . . . . . 39

2.1 Channel Estimation . . . . . . . . . . . . . . 40

2.2 Uplink Payload Transmission . . . . . . . . . . . . . 41

3 Sequential Uplink Processing . . . . . . . . . . . . . . . 42

3.1 Optimal Centralized Implementation . . . . . . . . . . 42

3.2 Sequential Linear Processing . . . . . . . . . . . . 43

$3.3 \quad$ Normalized LMMSE based sequential processing . . . 50

4 Fronthaul Signalling and Latency . . . . . . . . . . . . . 51

4.1 Centralized LMMSE Implementation . . . . . . . . . . 52

4.2 The OSLP Algorithm . . . . . . . . . . . . 52

4.3 Algorithm $3 \ldots \ldots \ldots \ldots \ldots \ldots$

4.4 The RLS Algorithm . . . . . . . . . . . . . 53

$4.5 \quad$ Latency . . . . . . . . . . . . . . . 53

$5 \quad$ Numerical Results and Discussions . . . . . . . . . . . 57

6 Conclusion . . . . . . . . . . . . . . . 62

7 Annex: Side Information in Sequential Processing . . . . . . . 67

B Distributed Computation of Bit Likelihood Ratios $\quad 71$

1 Introduction . . . . . . . . . . . . . . 73

2 System Model And Channel Estimation . . . . . . . . . . 75

2.1 Channel Estimation . . . . . . . . . . . . . 76

2.2 Uplink Payload Transmission . . . . . . . . . . . . . 77

3 Decentralized Detection . . . . . . . . . . . . . . . 77

3.1 MAP Detector for Hard Detection . . . . . . . . 78

3.2 Fronthaul signaling comparison . . . . . . . . . . . 80

3.3 LLR Calculation for Soft Detection . . . . . . . . . . 80

4 Conclusion . . . . . . . . . . . . . . 83 
C Energy-Efficient Power Allocation $\quad \mathbf{8 7}$

1 Introduction . . . . . . . . . . . . . . . . 89

2 System Model And Channel Estimation . . . . . . . . . . . 91

2.1 Channel Estimation . . . . . . . . . . . . . . . 92

2.2 Downlink Payload Transmission . . . . . . . . . . 93

3 Problem Formulation . . . . . . . . . . . . . . . . . . 95

4 Numerical Results . . . . . . . . . . . . . . . . . . . 98

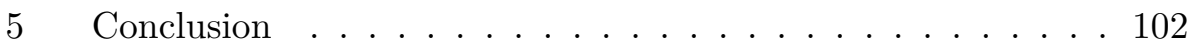




\section{Acknowledgements}

I want to extend my sincere thanks to my supervisors, Prof. Erik G. Larsson and Prof. Emil Björnson, for giving me the opportunity to work with one of the best research groups in the wireless communications research community. I am grateful to them for their valuable discussions on potential research directions, technical discussions, best practices in writing technical papers and help build rich research ethics. Thanks to Prof. Håkan Johansson, Asst. Prof. Zheng Chen and Snr. Lect. Dr. Mikael Olofsson for allowing me to be a teaching assistant in their courses which helped improve my teaching skills. I thank Docent. Danyo Danev for giving me the opportunity to supervise master student's thesis. I also thank Carina for all the help related to administrative work.

Since the time I have been in Sweden, most of whom I knew are my colleagues, and they are all more like good friends besides being colleagues, as they kept me grounded with life beyond work. I thank all my colleagues for creating a good research environment, especially my office-mate Ema, Ziya, Unnikrishnan, and Amin, for all the technical and non-technical discussions. I also like to thank Özgecan for being very helpful with teaching activities during my initial days at LiU. I also admire the time spent with Giovanni and Chien. I am thankful to Manoj and Sucharita for suggestions from their experiences on planning my PhD journey. I thank Sarvendra for the collaborative work during his time at LiU. I also thank Olle, Jianan, ChungHsuan, Ahmet, Oksana, Daniel, and Deijany for the good time spent.

I thank my friend Vivek for all the support during stressful work times. I thank all my friends and well-wishers from India, especially Gayatri, Sudeepini, Ravi, Kalyan, Pavani, Aishwarya, Vasu, Suman, Krishna, Sukesh, Anish, Kali, Ruchi, Anish, Nachiket, Prakash, Mrinalini and Heena, for being in regular contact and always wishing the best of me. Special thanks to my parents and brother, who always believed in and supported my research career path.

Zakir Hussain Shaik

Linköping, March 2022 


\section{List of Abbreviations}

$1 \mathrm{G} \quad$ first generation wireless technology

5G fifth generation wireless technology

6G sixth generation wireless technology

AWGN additive white Gaussian noise

BER bit error rate

CPU central processing unit

GSMA global system for mobile communications association

IP Internet Protocol

LLR log-likelihood ratio

MSE mean square error

MMSE minimum mean square error

MIMO multiple-input-multiple-output 



\section{Chapter 1}

\section{Introduction}

\subsection{Background and Motivation}

We live in a time when wireless devices have become ubiquitous around the globe. According to a recent report by GSMA [1], there are approximately 5.2 billion unique mobile users globally, accounting for about two-thirds of the world's population. This shows how much wireless communication has become such an integral part of everyone's life that an ordinary person cannot comprehend the ingenuity of work that goes behind it. From the evolutionary days of wireless radio, i.e., from the times of Nikolas Tesla and Guglielmo Marconi to recent days, wireless technology has evolved very rapidly. One of the most commercially successful and practical applications of wireless communication is cellular communications, pioneering technology for the vision of a globally connected world. Contemporary cellular networks were solely responsible for voice calls, however, now the world is witnessing an ever-increasing demand for internet data.

Although current cellular networks offer good data rates in the cell center, they suffer from large data rate variations within and at the cell edges. These non-uniform data rates make cellular services less reliable given the enormous data demands which continues to grow. Moreover, recently, more and more wireless applications are popping up like autonomous vehicles, navigation, and autonomous traffic systems, which demands the need to provide uniform quality of service in terms of data rate in the geographical coverage area. Towards this goal, there were attempts made by decreasing the cell-coverage area and deploying more access-points (APs, this is the term we would use in this thesis instead of conventional base station) per area unit [2,3]. The network capacity increases with the number of APs, but this trend gradually 
decreases and actually can reduce the capacity due to the increasing inter-cell interference [4]. Thus, further densification of the networks is not the solution for the long-term sustainability of wireless networks with current demands. There is a need for a paradigm shift in the way wireless networks operate, changing from traditional "cellular" networks to "cell-free" networks $[5,6]$. Thus, paving the way for cooperative operation of all the APs and jointly serving the user equipment (UEs) in the area by effectively mitigating the interference and increasing the system throughput. Towards this, Cell-Free Massive MIMO is envisaged to be a potential technology for beyond $5 \mathrm{G}$ and $6 \mathrm{G}$.

\subsection{Cell-Free Massive MIMO: A Blend of Best Existing Techniques}

The term Cell-Free Massive MIMO first appeared in [7]. However, the roots of the distributed cell-free system take inspiration from a conceptual architecture called Distributed Wireless Communication System [8]. The paper envisioned a network with distributed cooperating antennas, distributed processors, and distributed control protocols without "cell" boundaries. There are many other works that focused on cooperation of APs across multiple cells in serving the UEs and developing signal processing algorithms and communication protocols. These works have similar and overlapping ideas but with different terminologies, including, but not limited to, Group Cell [9, 10], Network MIMO [11-13], Cooperative Multi-Cell MIMO Networks [14-20], Coordinated Multipoint with Joint Processing (CoMP-JP) [21-32], Distributed Antenna System [29], Virtual MIMO [33], Extra Large Scale Massive MIMO [34], and Extremely Large Aperture Array $[35,36]$.

Conceptually, Cell-Free Massive MIMO is not a new concept but rather is an amalgamation of the following best existing techniques:

1. Physical layer aspects of cellular Massive MIMO:

- Massive number of antennas serving all the UEs in the geographical area, i.e., the total number of antennas serving UEs, is much larger than the number of UEs.

- Channel properties such as channel hardening and favorable propagation have an important consequence: linear processing provides close to optimal performance. The formal definitions of these terms are provided in Section 2.2.2. 
- The Time Division Duplex (TDD) operation enables the network to use the channel knowledge learned during uplink training phase in the downlink without having to re-estimate again. This is due to the channel property known as reciprocity.

2. CoMP-JP:

- Cooperation and distributed signal processing of cellular APs with partial or no channel state information (CSI) exchange between APs in both uplink and downlink.

- User-centric implementation where each UE selects a set of preferred APs. These sets are in general partially overlapping between neighboring UEs.

3. Ultra-Dense Networks: The large number of distributed antennas and ultra-densification of networks ensure each UE is surrounded by many APs thus providing enormous macro-diversity i.e., robustness against signal blockage as user-equipments (UEs) are highly likely to be close to some of the antennas.

4. Cell-Free architecture: a concept that envisions cell-free networks right from the beginning rather than deploying distributed signal processing on existing cellular networks.

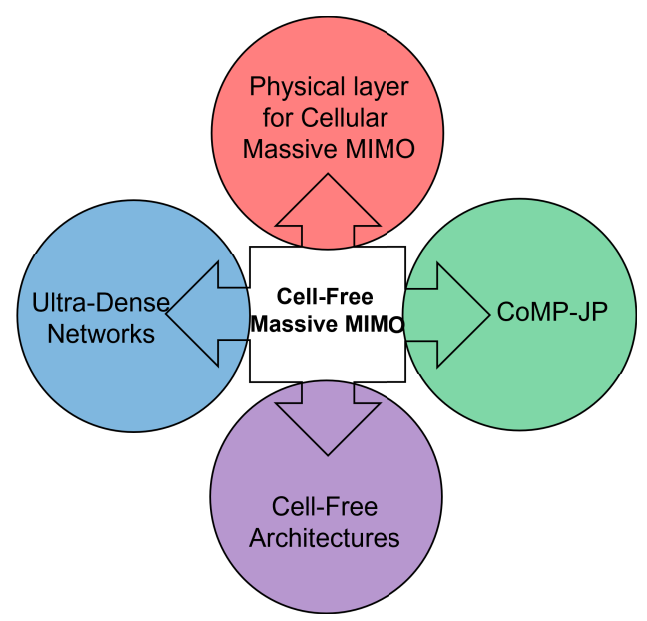

Figure 1: Describing Cell-Free Massive MIMO as a blend of four technologies. 
From the features shown in Figure 1, we can define Cell-Free Massive MIMO as an ultra-dense cell-free network operating in a TDD mode where the massive number of APs (all or a group) jointly and coherently serve all the UEs by exploiting the channel hardening and favorable propagation properties whenever applicable.

\subsubsection{Benefits of Cell-Free Massive MIMO}

We will now briefly cover the benefits of Cell-Free Massive MIMO under three broad categories:

- Macro-Diversity Gain: The ultra-dense deployment of APs ensures each UE sees at least one AP within the closest range. Numerical experiments have shown that the cell-free Massive MIMO offers higher and more uniform signal-to-interference-plus-noise-ratio (SINR) within the coverage area compared to Massive MIMO and small cells [37].

- Efficient Management of Interference: Cooperative implementation of APs helps in developing signal processing algorithms that can manage interference more effectively than networks with no cooperation (e.g., cellular networks and small cells).

- Beamforming and Multiplexing Gains: Cell-Free Massive MIMO inherits the high beamforming and multiplexing gains from Massive MIMO owning that there are many antennas distributed across the geographical area serving the UEs coherently.

\subsubsection{Topology of Cell-Free Massive MIMO}

The definition of Cell-Free Massive MIMO does not restrict the deployment architecture to a particular topology except that it should be distributed. The choice of specific hardware topology depends on the application, and the possible practical topologies include centralized, sequential (which we later call "radio stripe"), tree, and mesh. In this thesis we will consider a sequential topology owing to its benefits, such as less cabling requirement for implementation and ease of deployment. Distributed topology requires distributed signal processing, and most of the literature on distributed signal processing can be broadly categorized into four categories as indicated by different colors in Figure 2. Transition colors indicate there may be methods that may fall under a combination of these categories. The algorithms developed for first three categories can be extended and utilized in a CellFree Massive MIMO network. However, it depends on the ability of the 
algorithms to be decoupled and implemented on distributed hardware. In this thesis, all the proposed algorithms fall either in category 1 or 2 .

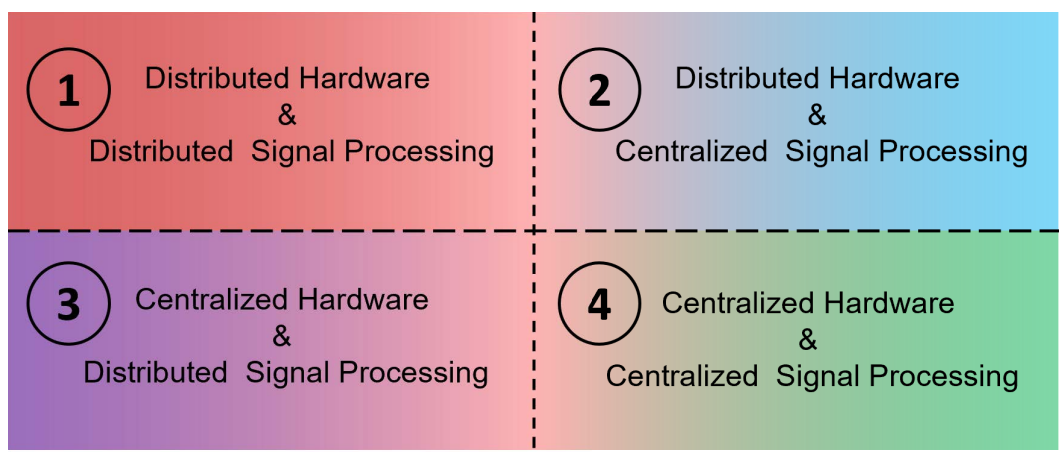

Figure 2: Taxonomy of Distributed Processing.

\subsection{Thesis Outline}

We dedicate Chapter 2 to building up the background for the included papers in the latter part of the thesis. We begin with the basic system model of wireless point-to-point communication, channel model and its characteristics, and performance metrics. Then using the theory developed, we will define the channel model for Cell-Free Massive MIMO and provide some of its properties. Chapter 2 also includes a basic definition and introduction to energy efficiency in wireless networks, which forms the background for Paper C.

Chapter 4 concisely puts the contributions of the thesis along with included and excluded papers. We end the first part of the thesis by providing concluding insights of the thesis in the form of a conclusion and future scope in Chapter 5. In the second part of the thesis, we provided the included refereed papers. 
1 Introduction 


\section{Chapter 2}

\section{Basics and Preliminaries}

\subsection{System Model}

Wireless channels are linear, thanks to Maxwell's equations. However, they generally are time-varying because the transmitter, receiver, and objects in and around can be mobile. Even the small movements (e.g., a few centimeters or even millimeters) will dramatically change the channel response. Nevertheless, if we consider a sufficiently small time interval, we can model the channel approximately as constant, enabling time-invariant wireless channel modeling. A basic point-to-point linear time-invariant (LTI) single-inputsingle-output (SISO) channel model consists of a single antenna transmitter and a single antenna receiver communicating over a wireless channel. A wireless communication channel acts like a filter that takes in the transmitted signal and outputs the received signal. The mathematical description of the SISO LTI model using complex baseband representation [38] is given below

$$
y=h x+n
$$

where $y$ is the received signal, $h$ is the channel response, $x$ is the transmitted signal and $n \sim \mathcal{C N}\left(0, \sigma^{2}\right)$ is the additive complex Gaussian noise with zero mean and variance $\sigma^{2}$.

The time interval during which the channel can be modeled as timeinvariant is called channel coherence time denoted by $T_{c}$. The factors that govern coherence time include mobility of transmitter, receiver, objects in between, and the operational frequency of the transmitted signal. The channel is considered as time-invariant if the receiver's relative motion does not change more than half-wavelength distance $\lambda / 2$ with relative velocity $v$ 
i.e.,

$$
T_{c}=\frac{\lambda}{2 v} .
$$

The channel can be modeled within the coherence time by a simple finite impulse response (FIR) filter [37]. Each term of the impulse response characterizes one distinct propagation path (described by distinct time delay and pathloss) in a multipath environment. Such modeling has a smoother variation of filter response with transmitted signal frequency. Moreover, if we consider a sufficiently narrow frequency interval, the channel can be considered approximately constant. The frequency range over which the frequency response is approximately constant is called as the channel coherence bandwidth denoted by $B_{c}$. The coherence bandwidth is dependent on the delay spread (approximate time difference between the arrival of earliest and the last multipath signal component) denoted by $T_{d}$ and they are related as follows:

$$
B_{c}=\frac{1}{T_{d}} .
$$

Remark 1: The ratio $f_{m}=v / \lambda$ is called as Doppler spread. Some textbooks, define coherence time as $T_{c}=\alpha \lambda / v$ with $0 \leq \alpha \leq 0.5$ and coherence bandwidth as $B_{c}=1 / 2 T_{d}[39,40]$.

\subsubsection{Coherence Block and Block Fading}

A channel coherence block (also called coherence interval) is a time-frequency space of duration $T_{c}$ seconds and bandwidth $B_{c} \mathrm{~Hz}$. When the channel between the transmitter and the receiver is said to be constant and frequency flat within a coherence block, then that means that the channel can be described by a single scalar constant (in general complex). A pictorial illustration of coherence block is shown in Figure. 3.

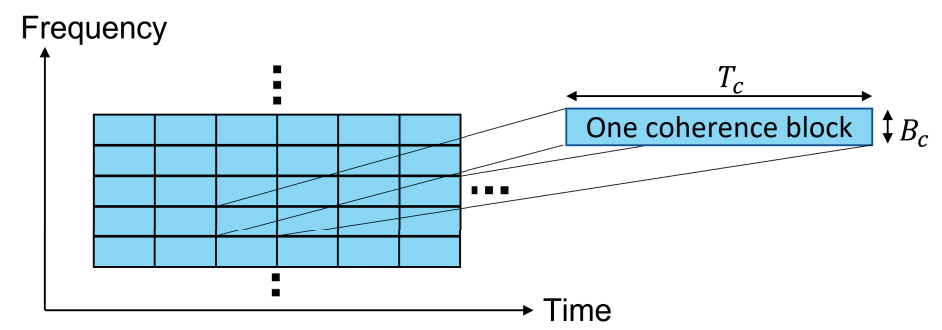

Figure 3: Depiction of time-frequency resources as coherence blocks. 
One theoretical benefit of modeling communication channels into coherence blocks is the convenience of developing signal processing algorithms as per coherence blocks and then analyze them separately. Furthermore, suppose the channel characterizing scalar constant takes an independent random realization in each coherence block, then without loss of generality, we can analyze one block at a time to describe the system performance. The channel fading model, which takes independent realization in each coherence block, is called the block-fading model. We assume in this thesis that the channel realizations between different coherence blocks are modeled as independent.

The relation between the coherence time and coherence bandwidth can be established using Nyquist-Shannon sampling theorem. According to the theorem, a signal that fits into coherence block i.e., a signal of duration $T_{c}$ seconds and bandwidth $B_{c} \mathrm{~Hz}$ (energy of the signal to a large extent contained in that frequency range), can be uniquely described by $\tau_{c}=T_{c} B_{c}$ complex samples. These $\tau_{c}$ samples can be used for channel estimation and payload transmission in uplink and downlink by duplex systems. The most commonly used protocols for channel estimation and payload transmissions are TDD and frequency-division duplexing (FDD). In this thesis, we focus only on the TDD protocol.

\subsubsection{Time-Division Duplexing Protocol}

In TDD operation, every coherence block is divided into three slots pictorially depicted in in Figure 4, where $\tau_{p}$ symbols are used for uplink pilots to estimate the channel, $\tau_{u}$ symbols are dedicated for uplink data, and $\tau_{d}$ symbols are assigned for downlink data such that $\tau_{c}=\tau_{p}+\tau_{u}+\tau_{d}$. An important advantage of TDD operation is that an AP can utilize the channel information learned in the uplink processing for downlink transmission due to channel reciprocity. All the work presented in this thesis assumes TDD operation.

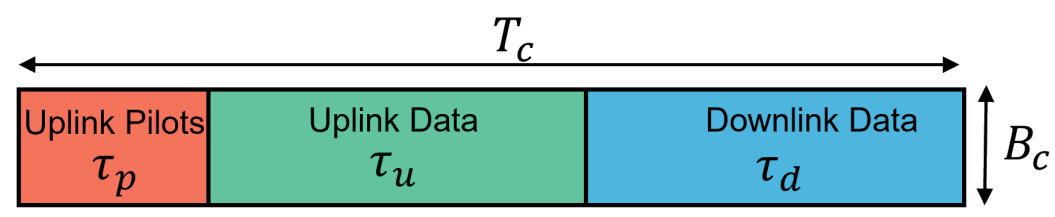

Figure 4: Depiction of time-frequency resources as coherence blocks. 


\subsubsection{Channel Model}

Ideally speaking, the wireless channel is deterministic. But, practically, it is impossible to account for all the possible multipath propagation between the transmitter and the receiver in modeling the channel. From the receiver's perspective, the channel variations appear randomly, thereby allowing the channel to be modeled both as stochastic and deterministic (in the scenarios where it is possible, e.g., free-space line-of-sight propagation). In this thesis, we focus on stochastic modeling of the channel. The received signal is the summation of many signal copies arriving with different path-loss and phase shifts. The resulting randomness at the receiver can be modeled as a Gaussian distribution due to the central limit theorem. The Gaussian distribution of the channel accounts for small-scale fading that accounts for small movements of the transmitter, receiver, or other objects in the environment. Mathematically, the Gaussian fading channel is denoted by $h \sim \mathcal{C N}(0, \beta)$. Here, $\beta$ is the large-scale fading coefficient taking into account the pathloss, shadowing and antenna gains. This channel model is more commonly called the Rayleigh fading channel since the magnitude of the scalar describing channel has a Rayleigh distribution.

Remark 2: The Rayleigh channel model is not just a theoretical model for comprehensive analysis of the wireless system but also practically viable. Practical measurements have shown that Rayleigh assumption is approximately correct for dense networks [41].

\subsubsection{Capacity Bounds as Performance Benchmark}

The ultimate aim of most of the signal processing algorithms employed in communication (wired or wireless) is to decode the transmitted signal successfully. To quantify the performance of the decoding algorithm, an appropriate benchmark is required. In this thesis, we consider channel capacity, besides MSE and BER as the performance metric. To briefly understand the concept of capacity, consider a simple channel model which takes in a scalar (complex-valued) input symbol $x$ and outputs a scalar (complex-valued) $y$. The transmitter maps the information bits to a sequence of symbols $\left\{x_{n}\right\}$, and the receiver applies signal processing algorithms on the received sequence of symbols $\left\{y_{n}\right\}$ and decodes the transmitted symbols (eventually bits). The effective number of bits transmitted per symbol is called the information rate or simply rate and is usually denoted by $R$ and is expressed in bits per channel use (bpcu). Transmitting a signal with a duration of $T$ seconds and bandwidth of $B \mathrm{~Hz}$ is equivalent to transmitting 
$B T$ symbols $\left\{x_{n}\right\}$ (this follows from Nyquist-Shannon sampling theorem). So in this context, the rate $R$ is sometimes called as spectral efficiency and is expressed in bits per second per Hertz $(\mathrm{b} / \mathrm{s} / \mathrm{Hz})$. Shannon-Hartley capacity theorem quantitatively expresses the maximum reliable rate called as capacity under a noisy channel model [42].

For most channel models, exact expression for the capacity is unknown. However, lower bounds on capacity exist, sometimes known as achievable rate or achievable spectral efficiency. We now present a generic lower bound on capacity [43, Sec 2.3.5] in Lemma 1, using which all the capacity bounds provided in this thesis can be derived.

Lemma 1: Consider a discrete memoryless channel with input symbol $x \in \mathbb{C}$, output symbol $y \in \mathbb{C}$, deterministic channel response $h \in \mathbb{C}$ and additive noise $w \in \mathbb{C}$ related as

$$
y=h x+w
$$

where we assume $\mathbb{E}\left\{|x|^{2}\right\} \leq p$ and $w$ has arbitrary distribution. We further assume that the receiver has access to side information $\Omega$ (possibly a set of random variables) and the following conditions hold

$$
\mathbb{E}\{w \mid \Omega\}=\mathbb{E}\left\{x^{*} w \mid \Omega\right\}=\mathbb{E}\left\{h^{*} x^{*} w \mid \Omega\right\}=0 .
$$

Then the lower-bound (achievable rate) on the capacity $C$ is as follows:

$$
C \geq \mathbb{E}\left\{\log _{2}\left(1+\frac{p|\mathbb{E}\{h \mid \Omega\}|^{2}}{p \operatorname{Var}\{h \mid \Omega\}+\mathbb{E}\left\{|w|^{2} \mid \Omega\right\}}\right)\right\}
$$

where the outer expectation is with respect to $\Omega$. Example: Let $h=\widehat{h}+\widetilde{h}$ and $\Omega=\{\widehat{h}, \widetilde{\beta}\}$, where $\widehat{h}$ is the channel estimate and $\widetilde{h} \sim \mathcal{C N}(0, \widetilde{\beta})$ is the channel estimation error which is Gaussian distributed with variance $\widetilde{\beta}$. We assume that: $\mathbb{E}\{w \mid \Omega\}=\mathbb{E}\left\{x^{*} w \mid \Omega\right\}=\mathbb{E}\left\{h^{*} x^{*} w \mid \Omega\right\}=0$ holds. Then the lower-bound is given by

$$
C \geq \mathbb{E}\left\{\log _{2}\left(1+\frac{p|\widehat{h}|^{2}}{p \widetilde{\beta}+\sigma^{2}}\right)\right\}
$$

where $\sigma^{2}=\mathbb{E}\left\{|w|^{2} \mid \Omega\right\}$ is the noise variance which is assumed to be known throughout this thesis. 


\subsection{Cell-Free Massive MIMO: Channel Model and Features}

\subsubsection{Spatially Correlated Channel Model}

Now we extend the basic system model described in 2.1 to Cell-Free Massive MIMO. We consider a system model comprising $L$ APs each equipped with $N$ antennas serving $K$ single antenna UEs. Let the channel between AP $l$ and UE $k$ is represented by a vector $\mathbf{h}_{k l}$

$$
\mathbf{h}_{k l} \sim \mathcal{C N}\left(\mathbf{0}, \mathbf{R}_{k l}\right)
$$

where $\mathbf{R}_{k l} \in \mathbb{C}^{N \times N}$ is the spatial correlation matrix between AP $l$ and UE $k$ which accounts for spatial channel correlation, shadowing, antenna gains, and pathloss.

We define the large-scale fading co-efficient as the average channel gain between AP $l$ and UE $k$ i.e.,

$$
\beta_{k l}=\frac{\operatorname{Tr}\left(\mathbf{R}_{k l}\right)}{N} .
$$

We assume throughout this thesis that channel vectors of different APs are uncorrelated i.e., $\mathbb{E}\left\{\mathbf{h}_{k l} \mathbf{h}_{k m}^{H}\right\}=\mathbf{0}_{N \times N}$ for $l \neq m$. This assumption is based on the premise that the APs are separated by tens if not hundreds of wavelengths.

Remark 3: The results generated in this thesis considered correlated Rayleigh fading. However, the theoretical framework can be extended to any channel model. The spatial correlation of the channel model is of practical importance for large antenna arrays because they have a good spatial resolution [39, Sec 2.2], which is one of the reason for choosing this model.

\subsubsection{Channel Hardening and Favorable Propagation}

The two key features of Massive MIMO, especially with the Rayleigh channel modeling, are channel hardening and favorable propagation. However, these properties do not clearly appear in Cell-Free Massive MIMO. Whether these properties appear or not in Cell-Free Massive MIMO depends on the number of antennas per AP and spatial channel properties. Since in Cell-Free Massive MIMO, each UE is served by multiple APs rather than a single cellular AP, we will define channel hardening and favorable propagation a little differently while retaining the underlying motivation [37, Sec 2.6]. The concept of 
channel hardening and favorable propagation can be well understood by considering the received signal at UE $k$ with a noiseless channel model given by

$$
\begin{aligned}
y_{k} & =\sum_{l=1}^{L} \mathbf{h}_{k l}^{H} \mathbf{x}_{l} \\
& =\sum_{l=1}^{L} \mathbf{h}_{k l}^{H} \mathbf{w}_{k l} s_{k}+\sum_{l=1}^{L} \sum_{i \neq k}^{K} \mathbf{h}_{k l}^{H} \mathbf{w}_{i l} s_{i}
\end{aligned}
$$

where $\mathbf{x}_{l}=\sum_{i=1}^{K} \mathbf{w}_{i l} s_{i}$ is the transmitted signal by AP $l, \mathbf{w}_{i l}$ is the precoder employed by AP $l$ for UE $i$, and $s_{i}$ is the independent signal intended for UE $i$ with $\mathbb{E}\left\{\left|s_{i}\right|^{2}\right\}=1$. The term $\sum_{l=1}^{L} \mathbf{h}_{k l}^{H} \mathbf{w}_{k l}$ is the effective channel and $\sum_{l=1}^{L} \sum_{i \neq k}^{K} \mathbf{h}_{k l}^{H} \mathbf{w}_{i l} s_{i}$ is the effective interference seen by UE $k$ due to concurrent transmission to other UEs.

\section{Channel Hardening}

Definition: In a Cell-Free Massive MIMO network the effective channel of UE $k$ is said to provide asymptotic channel hardening when the random realizations of the effective channel of UE $k$ are close to its mean value, i.e.,

$$
\frac{\sum_{l=1}^{L} \mathbf{h}_{k l}^{H} \mathbf{w}_{k l}}{\mathbb{E}\left\{\sum_{l=1}^{L} \mathbf{h}_{k l}^{H} \mathbf{w}_{k l}\right\}} \rightarrow 1 \text { as } N L \rightarrow \infty
$$

The term harden refers to the diminishing randomness of the effective channel and becoming more deterministic. Strictly speaking, the denominator in (11) can be any deterministic non-zero constant for definition of channel hardening to hold, however, with most of the wireless channel models used in literature the effective channel converges to the mean value due to the law of large numbers.

In literature, it has been a convention to define the channel hardening using the maximum ratio (MR) precoder. This is primarily done because the choice of MR precoder makes analysis of channel hardening tractable and provides useful insights into the system's performance. Following are two examples defined for Cell-Free Massive MIMO and cellular Massive MIMO, respectively:

- For Cell-Free Massive MIMO [37, Sec 2.6.1]: Set the precoder $\mathbf{w}_{k l}=$ $\sqrt{\frac{p_{k l}}{\mathbb{E}\left\{\left\|\mathbf{h}_{k l}\right\|^{2}\right\}}} \mathbf{h}_{k l}$ where $p_{k l}>0$ (active user) is the power allocated to UE $k$ by AP $l$. Then for a given $L$, the effective channel seen by UE $k$ is 
said to achieve asymptotic channel hardening if

$$
\frac{\sum_{l=1}^{L} \sqrt{\frac{p_{k l}}{\mathbb{E}\left\{\left\|\mathbf{h}_{k l}\right\|^{2}\right\}}}\left\|\mathbf{h}_{k l}\right\|^{2}}{\mathbb{E}\left\{\sum_{l=1}^{L} \sqrt{\frac{p_{k l}}{\mathbb{E}\left\{\left\|\mathbf{h}_{k l}\right\|^{2}\right\}}}\left\|\mathbf{h}_{k l}\right\|^{2}\right\}} \rightarrow 1
$$

in the mean-squared sense as $N \rightarrow \infty$ [37]. Since, one of the most common wireless channel model is Rayleigh, it is useful to provide conditions on which the channel hardening is achieved. With correlated Rayleigh fading, a sufficient condition for channel hardening for the effective channel at UE $k$ to provide asymptotic channel hardening is

$$
\frac{\sum_{l=1}^{L} p_{k l} \frac{\operatorname{Tr}\left(\mathbf{R}_{k l}^{2}\right)}{N \beta_{k l}}}{N\left(\sum_{l=1}^{L} \sqrt{p_{k l} \beta_{k l}}\right)^{2}} \rightarrow 0 \text { as } N \rightarrow \infty .
$$

- For Massive MIMO [43, Sec 7.2.1]: Set $L=1, M=N L, \mathbf{R}_{k}=\beta_{k} \mathbf{I}_{M}$ (dropping index $l$ ) and $\mathbf{w}_{k}=\sqrt{p_{k}} \frac{\mathbf{h}_{k}}{M}, p_{k}>0$. Assuming $\beta_{k}$ is finite, the channel hardening condition (11) becomes

$$
\frac{\left\|\mathbf{h}_{k}\right\|^{2}}{M} \rightarrow \beta_{k} \text { as } M \rightarrow \infty
$$

\section{Favorable Propagation}

When multiple UEs are spatially multiplexed in the system, there is inherent inter-user interference. From (10), it is desirable for UE $k$ to have channel conditions such that system design can exploit the properties of the channel and completely mitigate the interference caused by concurrent transmission to other UEs. Accordingly, we say that the UE $k$ experiences asymptotic desirable propagation, if the effective interference vanishes with asymptotic increase in the total number of antennas i.e.,

$$
\sum_{l=1}^{L} \mathbf{h}_{k l}^{H} \mathbf{w}_{i l} \rightarrow 0 \text { as } N L \rightarrow \infty, \forall i \in\{1, \ldots, K\} \backslash\{k\} .
$$

Moreover, the UE $k$ experiences the most favorable situation when all the UE channels are orthogonal to each other because then the interference can be mitigated completely by simple MR precoder (e.g., $\mathbf{w}_{i l}=\sqrt{p_{i l}} \frac{\mathbf{h}_{i l}}{N L}, p_{i l}>0$ ). When channels are orthogonal we say that the system experiences favorable propagation. We define favorable propagation slightly different than 
commonly used in the literature by taking motivation from the definition presented in [37, Sec 2.6.2].

Definition: In a Cell-Free Massive MIMO networks we say that UE $k$ experiences asymptotically favorable propagation for given $p_{i l}>0$, if the following condition holds

$$
\frac{\sum_{l=1}^{L} \sqrt{p_{i l}} \mathbf{h}_{k l}^{H} \mathbf{h}_{i l}}{N L} \rightarrow 0 \text { as } N L \rightarrow \infty, \forall i \in\{1, \ldots, K\} \backslash\{k\} .
$$

The following are, two of the consequences of the definition in (16)

- One of the sufficient conditions for (16) to hold is

$$
\frac{\mathbf{h}_{k l}^{H} \mathbf{h}_{i l}}{N} \rightarrow 0 \text { as } N \rightarrow \infty, \forall i \in\{1, \ldots, K\} \backslash\{k\}, \forall l \in\{1, \ldots, L\} .
$$

The above condition precisely means that the channels of different users at a given AP are asymptotically orthogonal. The condition in (17) will hold for uncorrelated channels and sufficiently large antennas at each AP.

- The favorable propagation definition for Massive MIMO [43, Sec 7.2.1]: Set $L=1, M=N L, \mathbf{R}_{k}=\beta_{k} \mathbf{I}_{M}$ (dropping index $l$ ) and $\mathbf{w}_{i}=\sqrt{p_{i}} \frac{\mathbf{h}_{i}}{M}$. Then the favorable condition in (16) becomes

$$
\frac{\mathbf{h}_{k}^{H} \mathbf{h}_{i}}{M} \rightarrow 0 \text { as } M \rightarrow \infty, \forall i \in\{1, \ldots, K\} \backslash\{k\} .
$$

\section{Some remarks on channel hardening and favorable propagation}

- The definitions of channel hardening and favorable propagation is presented from downlink perspective. However, similar argumentation can be made in the uplink (with centralized implementation), by replacing precoding vectors with combining vectors, and the power allocation coefficients with weights that the CPU employs for fusing the signal estimates from all the APs.

- These properties, if applicable, are especially useful in downlink communication by effectively turning the fading channel into an approximate AWGN channel. However, in uplink the APs have access to the instantaneous channel (from the channel estimates) and therefore have more information about the effective channel than just its statistics. 
- One of the most common capacity lower bounds used in cellular Massive MIMO and Cell-Free Massive MIMO literature is the use-and-thenforget bound, also called the hardening bound [37,43], is based on the channel hardening definition in (11).

- These properties were discussed in this section to formally define these concepts in the context of Cell-Free Massive MIMO and connect them with the definitions of Massive MIMO. However, the results presented in the included papers in later part of this thesis do not formally need these properties to hold.

- Although all the definitions presented rely on asymptotic arguments, these definitions can be evaluated for practical purpose with sufficiently large number of antennas, for example as done in [37]. Exact asymptotic convergence type (e.g., convergence in probability) is not important because deployment of infinite antennas is impractical and there are limitation posed by physics, for more details refer to [44].

- In [37], authors observed that:

- The spatial correlation of channels reduces the convergence rate of the channel hardening than with uncorrelated channels.

- With increase in $N$ the effective channel achieves faster channel hardening convergence than when increasing $L$.

\section{$2.3 \quad$ Energy Efficiency}

\subsubsection{Motivation and Definition}

Over the past few years, the dramatic increase in the number of wireless devices poses a challenging question for the goal of global sustainable growth. For instance, it is predicted that the global consumer IP traffic will reach 332.7 exabytes this year, i.e., 2022 [45] which is more than four times the traffic in 2015 [46]. According to an another finding the information and communication technology (ICT) accounted for $1.15 \%$ of the total global electricity grid supply in 2015 [47]. These reports roughly indicate that the energy consumption of the ICT may grow proportionally to traffic if proper measures are not taken as most internet data reception these days is through wireless devices. Thus, there is an increasing demand for energyefficient devices and signal processing algorithms to cater to current and future demands. 
One of the standard performance metrics to quantitatively evaluate the system performance concerning energy consumed in wireless networks is energy efficiency (EE). The EE of a wireless network is defined as the number of bits that can be reliably transmitted per unit of energy i.e.,

$$
\mathrm{EE}=\frac{\text { Throughput }[\mathrm{bit} / \mathrm{s}]}{\text { Power consumption }[\mathrm{W}]}
$$

which is measured in bit/Joule. The throughput is computed using spectral efficiency expressions of the uplink or downlink model, sometimes combining both expressions. There are various definitions of EE in the literature [48], but we use a particular one in which throughput is expressed in terms of achievable sum-rate. Detailed analysis is done in Paper C.

When designing signal processing algorithms that focus on maximizing the EE of the network with certain constraints such as limited power budget, one often deals with fractional problems. We will present one particular case of fractional problems, which is useful in understanding the algorithm proposed in Paper C.

\subsubsection{Dinkelbach's Algorithm}

Let us consider the following optimization problem:

$$
\begin{aligned}
\underset{\mathbf{x}}{\operatorname{maximize}} & \frac{f(\mathbf{x})}{g(\mathbf{x})}, \\
\text { s.t. } & c_{i}(\mathbf{x}) \leq 0, \forall i=1, \ldots, m \\
& h_{j}(\mathbf{x})=0, \forall j=1, \ldots, n
\end{aligned}
$$

where $g(\mathbf{x})$ and $c_{i}(\mathbf{x})$ are convex, $f(\mathbf{x})$ is concave and $h_{j}(\mathbf{x})$ are affine.

The problem (20) belongs to the broad class of fractional programming, and there exist many algorithms to solve it. We in this thesis will use the algorithm known as Dinkelbach's algorithm which falls under the category of parametric algorithms. The algorithm involves solving a series of subproblems which eventually converges to the global solution of (20). The basic working principle of the algorithm is based on the relation between the (20) and the following function

$$
F(\lambda)=\underset{\mathbf{x} \in \mathcal{S}}{\operatorname{maximize}}\{f(\mathbf{x})-\lambda g(\mathbf{x})\}
$$

where the $\mathcal{S}$ is the feasibility set of (20). Few of the interesting properties of $F(\lambda)$ when we assume $f(\mathbf{x})$ and $g(\mathbf{x}) \geq 0$ are continuous, and $\mathcal{S}$ is compact 
are: (i) $F(\lambda)$ exists and is continuous, (ii) $F(\lambda)$ is convex on $\mathbb{R}$, (iii) $F(\lambda)$ decreases monotonically on $\mathbb{R}$, and $(i v) F(\lambda)$ has a unique root. Besides these properties the most important feature is as follows: consider $\mathrm{x}^{*} \in \mathcal{S}$ and define $\lambda^{*}=\frac{f\left(\mathbf{x}^{*}\right)}{g\left(\mathbf{x}^{*}\right)}$, then $\mathbf{x}^{*}$ is the optimal solution of (20) if and only if

$$
\mathbf{x}^{*}=\underset{\mathbf{x} \in \mathcal{S}}{\operatorname{argmax}}\left\{f(\mathbf{x})-\lambda^{*} g(\mathbf{x})\right\} .
$$

For proof we refer to [49]. The overall algorithm is presented in Algorithm 5.

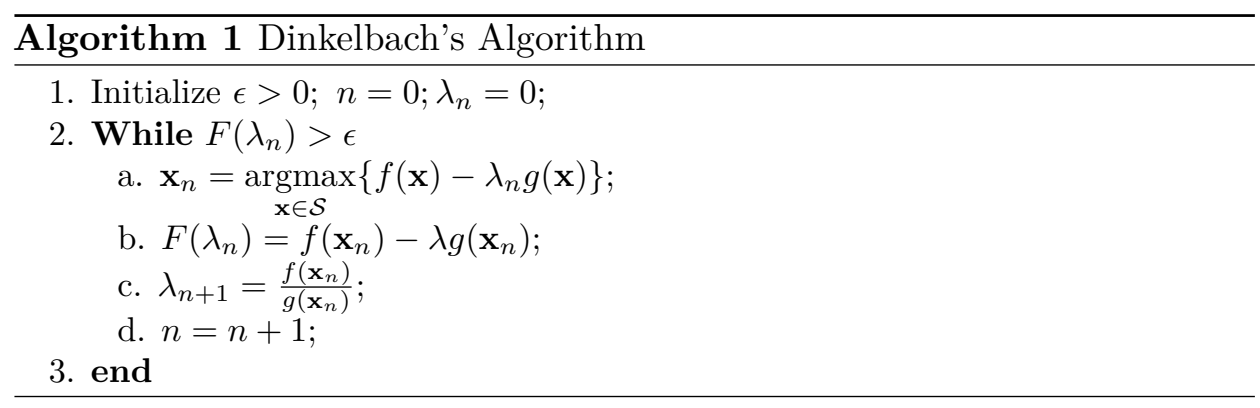

The Dinkelbach's algorithm has superlinear convergence rate in the subproblem sequence and computational complexity of sub-problem is polynomial in the number of variables and constraints [49]. 


\section{Chapter 3}

\section{Contributions of the Thesis}

This thesis focuses on the distributed signal processing algorithms in CellFree Massive MIMO. In Paper A we developed a general linear distributed processing for radio stripes, and specifically, we proposed an optimal linear MMSE algorithm motivated from Kalman filtering. In Paper B, we developed a distributed algorithm to compute the bit LLR for radio stripes. Finally, Paper $\mathrm{C}$ proposed an algorithm for maximizing the energy efficiency of the RadioWeaves network with underlay spectrum sharing. RadioWeaves is an emerging technology built upon the concepts of Cell-Free Massive MIMO and possibly large intelligent surfaces wherein the antennas and underlying signal processing circuitry are weaved into large surface areas such as conventional buildings and objects [50].

\subsection{Included Papers}

\section{Paper A: MMSE-Optimal Sequential Processing for Cell-Free Massive MIMO With Radio Stripes}

Authored by: Zakir Hussain Shaik, Emil Björnson, and Erik G. Larsson Published in IEEE Transactions on Communications, vol. 69, no. 11, pp. 7775-7789, Nov. 2021.

Abstract: Cell-free massive multiple-input-multiple-output (mMIMO) is an emerging technology for beyond $5 \mathrm{G}$ with its promising features such as higher spectral efficiency and superior spatial diversity as compared to conventional multiple-input- multiple-output (MIMO) technology. The main working principle of cell-free mMIMO is that many distributed access points (APs) cooperate simultaneously to serve all the users within the network 
without creating cell boundaries. This paper considers the uplink of a cellfree mMIMO system utilizing the radio stripe network architecture with a sequential fronthaul between the APs. A novel uplink sequential processing algorithm is developed, which is proved to be optimal in both the maximum spectral efficiency (SE) and the minimum MSE sense. A detailed quantitative analysis of the fronthaul requirement or signaling of the proposed algorithm and its comparison with competing sub-optimal algorithms is provided. Key conclusions and implications are summarized in the form of corollaries. Based on the analytical and numerical simulation results, we conclude that the proposed scheme can significantly reduce the fronthaul signaling, without compromising the communication performance.

\title{
Paper B: Distributed Computation of A Posteriori Bit Likelihood Ratios in Cell-Free Massive MIMO
}

Authored by: Zakir Hussain Shaik, Emil Björnson, and Erik G. Larsson

Published in the proceedings of 2021 29th European Signal Processing (EUSIPCO) Conference

\begin{abstract}
This paper presents a novel strategy to decentralize the soft detection procedure in an uplink cell-free massive multiple-input-multipleoutput network. We propose efficient approaches to compute the a posteriori probability-per-bit, exactly or approximately, when having a sequential fronthaul. More precisely, each access point (AP) in the network computes partial sufficient statistics locally, fuses it with received partial statistics from another AP, and then forward the result to the next AP. Once the sufficient statistics reach the central processing unit, it performs the soft demodulation by computing the log-likelihood ratio (LLR) per bit, and then a channel decoding algorithm (e.g., a Turbo decoder) is utilized to decode the bits. We derive the distributed computation of LLR analytically.
\end{abstract}




\section{Paper C: Energy-Efficient Power Allocation for an Underlay Spectrum Sharing RadioWeaves Network}

Authored by: Zakir Hussain Shaik, Rimalapudi Sarvendranath, and Erik G. Larsson

Accepted for publication in the proceedings of 2022 IEEE International Conference on Communications (ICC) Conference

Abstract: RadioWeaves network operates a large number of distributed antennas using cell-free architecture to provide high data rates and support a large number of users. Operating this network in an energy-efficient manner in the limited available spectrum is crucial. Therefore, we consider energy efficiency (EE) maximization of a RadioWeaves network that shares spectrum with a collocated primary network in underlay mode. To simplify the problem, we lower bound the non-convex EE objective function to form a convex problem. We then propose a downlink power allocation policy that maximizes the EE of the secondary RadioWeaves network subject to power constraint at each access point and interference constraint at each primary user. Our numerical results investigate the secondary system's performance in interference, power, and EE constrained regimes with correlated fading channels. Furthermore, they show that the proposed power allocation scheme performs significantly better than the simpler equal power allocation scheme.

\subsection{Excluded Papers}

The following paper is excluded from the thesis due to it being superfluous.

\section{Cell-Free Massive MIMO With Radio Stripes and Sequential Uplink Processing}

Authored by: Zakir Hussain Shaik, Emil Björnson, and Erik G. Larsson

Published in the proceedings of IEEE International Conference on

Communications Workshops (ICC Workshops), 2020, pp. 1-6

This paper contains preliminary results of Paper A.

Abstract: Cell-free Massive MIMO (mMIMO) is envisaged to be a nextgeneration technology beyond $5 \mathrm{G}$ with its high spectral efficiency and superior spatial diversity as compared to that of conventional MIMO technology. The main principle is that many distributed access points (APs) cooperate to simultaneously serve all the users within the network without creating cell boundaries. This paper considers the uplink of a cell-free mMIMO system utilizing the radio stripe network architecture. We propose a novel sequential 
processing algorithm with normalized linear minimum mean square error (N-LMMSE) combining at every AP. This algorithm enables interference suppression in cell-free mMIMO while keeping the cost and front-haul requirements low. The spectral efficiency of the proposed algorithm is computed and analyzed. We conclude that it provides an attractive trade-off between low front-haul requirements and high spectral efficiency. 


\section{Chapter 4}

\section{Conclusion and Future Scope}

Cell-Free Massive MIMO promises uniform quality of service and connectivity to all the users in its geographical coverage area thanks to its attractive features presented in Section 1.2.1 such as micro-diversity. For Cell-Free Massive MIMO to be practically deployed and avail its potential benefits, there is a need for distributed topologies with practical viability. Among various distributed architectures, we consider sequentially connected APs mainly for their ease of deployment.

In this thesis, we first focused on developing optimal algorithms in the sense of MSE and bit log-likelihood computation for sequentially deployed Cell-Free Massive MIMO. We showed that there is no loss in performance when compared to a centralized Cell-Free Massive MIMO. Moreover, with the proposed algorithms, the fronthaul (number of real symbols required by the CPU to decode the transmitted signal) of the network is independent of the number of APs. In Paper A, we developed a generic linear signal processing framework for a sequential network and then proposed an algorithm that is optimal in both MSE and SE. The proposed algorithm is analytically equivalent to centralized implementation but with significantly less fronthaul requirement. In Paper B, we proposed an algorithm to compute the centralized bit-LLR in a distributed sequential network while keeping fronthaul requirement independent of the number of APs.

In Cell-Free Massive MIMO, there are many distributed antennas serving UEs, thereby making the energy efficiency of the network of paramount importance. Keeping this in mind, we proposed an iterative algorithm in Paper C. The proposed algorithm is dependent only on the channel statistics, and there is no requirement for sharing any instantaneous CSI information among APs. Thus, this algorithm is suitable for sequential and any distributed 
topology as, ideally, the statistics are fixed for the setup and the system design parameters.

This thesis assumes that UEs have a single antenna and sequential signal processing for multi-antenna users is an open problem. The channel model considered is the correlated Rayleigh fading; one can explore the design of algorithms for the Rician model, which also considers the direct signal path between transmitter and receiver, such as line-of-sight. Nevertheless, the framework developed in the thesis can be extended to other channel models. The energy efficiency problem presented in Paper $\mathrm{C}$ is non-convex and we developed an algorithm for the convex lower bound on the original non-convex problem. Therefore, the algorithm proposed is a sub-optimal solution. So, there is a window to improve upon the algorithm to gain further without increasing the complexity. 


\section{Bibliography}

[1] K. Okeleke and S. Suardi, "The mobile economy 2021," GSMA, Tech. Rep., 2021. [Online]. Available: https://www.gsma.com/mobileeconomy/ wp-content/uploads/2021/07/GSMA_MobileEconomy2021_3.pdf

[2] M. Kamel, W. Hamouda, and A. Youssef, "Ultra-dense networks: A survey," IEEE Communications Surveys Tutorials, vol. 18, no. 4, pp. 2522-2545, Fourthquarter 2016.

[3] S. Stefanatos and A. Alexiou, "Access point density and bandwidth partitioning in ultra dense wireless networks," IEEE Transactions on Communications, vol. 62, no. 9, pp. 3376-3384, Sep. 2014.

[4] J. G. Andrews, X. Zhang, G. D. Durgin, and A. K. Gupta, "Are we approaching the fundamental limits of wireless network densification?" IEEE Communications Magazine, vol. 54, no. 10, pp. 184-190, Oct 2016.

[5] J. Zhang, S. Chen, Y. Lin, J. Zheng, B. Ai, and L. Hanzo, "Cell-free massive MIMO: A new next-generation paradigm," IEEE Access, vol. 7, pp. $99878-99888,2019$.

[6] G. Interdonato, E. Björnson, H. Q. Ngo, P. Frenger, and E. G. Larsson, "Ubiquitous cell-free massive MIMO communications," EURASIP Journal on Wireless Communications and Networking, vol. 2019, no. 1, p. 197, 2019.

[7] H. Q. Ngo, A. Ashikhmin, H. Yang, E. G. Larsson, and T. L. Marzetta, "Cell-free massive MIMO versus small cells," IEEE Transactions on Wireless Communications, vol. 16, no. 3, pp. 1834-1850, Mar 2017.

[8] S. Zhou, M. Zhao, X. Xu, J. Wang, and Y. Yao, "Distributed wireless communication system: a new architecture for future public wireless access," IEEE Communications Magazine, vol. 41, no. 3, pp. 108-113, Mar 2003.

[9] P. Zhang, X. Tao, J. Zhang, Y. Wang, L. Li, and Y. Wang, "A vision from the future: beyond 3G TDD," IEEE Communications Magazine, vol. 43, no. 1, pp. 38-44, Jan 2005.

[10] X. Tao, J. Xu, X. Xu, C. Tang, and P. Zhang, "Group cell FuTURE B3G TDD system," in IEEE 16th International Symposium on Personal, Indoor and Mobile Radio Communications, vol. 2, Sep. 2005, pp. 967-971. 
[11] S. Venkatesan, A. Lozano, and R. Valenzuela, "Network MIMO: Overcoming intercell interference in indoor wireless systems," in Conference Record of the Forty-First Asilomar Conference on Signals, Systems and Computers, Nov 2007, pp. 83-87.

[12] G. Caire, S. A. Ramprashad, and H. C. Papadopoulos, "Rethinking network MIMO: Cost of CSIT, performance analysis, and architecture comparisons," in Information Theory and Applications Workshop (ITA), Jan 2010, pp. 1-10.

[13] I. D. Garcia, N. Kusashima, K. Sakaguchi, and K. Araki, "Dynamic cooperation set clustering on base station cooperation cellular networks," in 21st Annual IEEE International Symposium on Personal, Indoor and Mobile Radio Communications, Sep. 2010, pp. 2127-2132.

[14] A. Papadogiannis, D. Gesbert, and E. Hardouin, "A dynamic clustering approach in wireless networks with multi-cell cooperative processing," in IEEE International Conference on Communications, May 2008, pp. 4033-4037.

[15] S. Shamai and B. Zaidel, "Enhancing the cellular downlink capacity via co-processing at the transmitting end," in IEEE VTS 53rd Vehicular Technology Conference, Spring 2001. Proceedings (Cat. No.01CH37202), vol. 3, May 2001, pp. 1745-1749.

[16] S. Jafar, G. Foschini, and A. Goldsmith, "Phantomnet: exploring optimal multicellular multiple antenna systems," in Proceedings IEEE 56th Vehicular Technology Conference, vol. 1, Sep 2002, pp. 261-265.

[17] H. Zhang and H. Dai, "Cochannel interference mitigation and cooperative processing in downlink multicell multiuser MIMO networks," EURASIP Journal on Wireless Communications and Networking, vol. 2004, no. 2, pp. 1-14, 2004.

[18] G. Foschini, K. Karakayali, and R. Valenzuela, "Coordinating multiple antenna cellular networks to achieve enormous spectral efficiency," IEE Proceedings-Communications, vol. 153, no. 4, pp. 548-555, 2006.

[19] M. Karakayali, G. Foschini, and R. Valenzuela, "Network coordination for spectrally efficient communications in cellular systems," IEEE Wireless Communications, vol. 13, no. 4, pp. 56-61, Aug 2006. 
[20] E. Björnson, R. Zakhour, D. Gesbert, and B. Ottersten, "Cooperative multicell precoding: Rate region characterization and distributed strategies with instantaneous and statistical CSI," IEEE Transactions on Signal Processing, vol. 58, no. 8, pp. 4298-4310, Aug 2010.

[21] R. Irmer, H. Droste, P. Marsch, M. Grieger, G. Fettweis, S. Brueck, H.-P. Mayer, L. Thiele, and V. Jungnickel, "Coordinated multipoint: Concepts, performance, and field trial results," IEEE Communications Magazine, vol. 49, no. 2, pp. 102-111, Feb 2011.

[22] P. Marsch and G. P. Fettweis, Coordinated Multi-Point in Mobile Communications: From Theory to Practice. Cambridge University Press, 2011.

[23] V. Jungnickel, K. Manolakis, W. Zirwas, B. Panzner, V. Braun, M. Lossow, M. Sternad, R. Apelfröjd, and T. Svensson, "The role of small cells, coordinated multipoint, and massive MIMO in 5G," IEEE Communications Magazine, vol. 52, no. 5, pp. 44-51, May 2014.

[24] F. Irram, M. Ali, Z. Maqbool, F. Qamar, and J. J. Rodrigues, "Coordinated multi-point transmission in $5 \mathrm{G}$ and beyond heterogeneous networks," in IEEE 23rd International Multitopic Conference (INMIC), Nov 2020, pp. 1-6.

[25] S. Parkvall, E. Dahlman, A. Furuskär, Y. Jading, M. Olsson, S. Wänstedt, and K. Zangi, "LTE-advanced - evolving LTE towards IMT-advanced," in IEEE VTC-Fall, 2008.

[26] H. Huang, M. Trivellato, A. Hottinen, M. Shafi, P. Smith, and R. Valenzuela, "Increasing downlink cellular throughput with limited network MIMO coordination," vol. 8, no. 6, pp. 2983-2989, 2009.

[27] P. Marsch and G. Fettweis, "On multicell cooperative transmission in backhaul-constrained cellular systems," Ann. Telecommun., vol. 63, pp. 253-269, 2008.

[28] J. Zhang, R. Chen, J. G. Andrews, A. Ghosh, and R. Heath, "Networked MIMO with clustered linear precoding," vol. 8, no. 4, pp. 1910-1921, 2009 .

[29] W. Choi and J. G. Andrews, "Downlink performance and capacity of distributed antenna systems in a multicell environment," vol. 6, no. 1, pp. 69-73, Jan 2007. 
[30] E. Björnson and E. Jorswieck, "Optimal resource allocation in coordinated multi-cell systems," Foundations and Trends® in Communications and Information Theory, vol. 9, no. 2-3, pp. 113-381, 2013.

[31] S. Chen, F. Qin, B. Hu, X. Li, and Z. Chen, "User-centric ultra-dense networks for 5G: challenges, methodologies, and directions," IEEE Wireless Communications, vol. 23, no. 2, pp. 78-85, April 2016.

[32] S. Kaviani, O. Simeone, W. Krzymien, and S. Shamai, "Linear precoding and equalization for network MIMO with partial cooperation," vol. 61, no. 5, pp. 2083-2095, 2012.

[33] W. Feng, Y. Wang, N. Ge, J. Lu, and J. Zhang, "Virtual MIMO in multi-cell distributed antenna systems: Coordinated transmissions with large-scale CSIT," IEEE Journal on Selected Areas in Communications, vol. 31, no. 10, pp. 2067-2081, Oct 2013.

[34] E. D. Carvalho, A. Ali, A. Amiri, M. Angjelichinoski, and R. W. Heath, "Non-stationarities in extra-large-scale massive MIMO," IEEE Wireless Communications, vol. 27, no. 4, pp. 74-80, Aug 2020.

[35] E. Björnson, L. Sanguinetti, H. Wymeersch, J. Hoydis, and T. L. Marzetta, "Massive MIMO is a reality - what is next?: Five promising research directions for antenna arrays," Digital Signal Processing, vol. 94, pp. 3 - 20, 2019, special Issue on Source Localization in Massive MIMO. [Online]. Available: http://www.sciencedirect.com/science/article/pii/S1051200419300776

[36] A. Amiri, M. Angjelichinoski, E. de Carvalho, and R. W. Heath, "Extremely large aperture massive MIMO: Low complexity receiver architectures," in Proc. IEEE GLOBECOM Workshops, 2018.

[37] Ö. T. Demir, E. Björnson, and L. Sanguinetti, "Foundations of usercentric cell-free massive MIMO," Foundations and Trends in Signal Processing, vol. 14, no. 3-4, pp. 162-472, 2021.

[38] D. Tse and P. Viswanath, Fundamentals of wireless communication. Cambridge University Press, 2005.

[39] E. Björnson, J. Hoydis, and L. Sanguinetti, "Massive MIMO networks: Spectral, energy, and hardware efficiency," Foundations and Trends® in Signal Processing, vol. 11, no. 3-4, pp. 154-655, 2017. [Online]. Available: http://dx.doi.org/10.1561/2000000093 
[40] A. Goldsmith, Wireless communications. Cambridge university press, 2005 .

[41] D. Chizhik, J. Ling, P. Wolniansky, R. Valenzuela, N. Costa, and K. Huber, "Multiple-input-multiple-output measurements and modeling in Manhattan," IEEE Journal on Selected Areas in Communications, vol. 21, no. 3, pp. 321-331, April 2003.

[42] C. E. Shannon, "A mathematical theory of communication," The Bell System Technical Journal, vol. 27, no. 3, pp. 379-423, July 1948.

[43] T. L. Marzetta, E. G. Larsson, H. Yang, and H. Q. Ngo, Fundamentals of Massive MIMO. Cambridge University Press, 2016.

[44] E. Björnson and L. Sanguinetti, "Power scaling laws and near-field behaviors of massive MIMO and intelligent reflecting surfaces," IEEE Open Journal of the Communications Society, vol. 1, pp. 1306-1324, 2020 .

[45] CISCO, "VNI complete forecast highlights," Tech. Rep., 2018. [Online]. Available: https://www.cisco.com/c/dam $/ \mathrm{m} / \mathrm{en}$ us/solutions/service-provider/vni-forecast-highlights/pdf/Global_ Device_Growth_Traffic_Profiles.pdf

[46] — , "VNI complete forecast highlights," Tech. Rep., 2015. [Online]. Available: https://www.cisco.com/c/dam/m/en_us/solutions/ service-provider/vni-forecast-highlights/pdf/Global_2015_Year_in_ Review.pdf

[47] J. Malmodin and D. Lundén, "The electricity consumption and operational carbon emissions of ICT network operators 2010-2015," KTH, Centre for Sustainable Communications, CESC, Tech. Rep. 2018:1, QC 20180125 .

[48] D. López-Pérez, A. De Domenico, N. Piovesan, G. Xinli, H. Bao, S. Qitao, and M. Debbah, "A survey on $5 \mathrm{G}$ radio access network energy efficiency: Massive MIMO, lean carrier design, sleep modes, and machine learning," IEEE Communications Surveys Tutorials, pp. 1-1, 2022.

[49] A. Zappone and E. Jorswieck, "Energy efficiency in wireless networks via fractional programming theory," Foundations and Trends in Communications and Information Theory, vol. 11, no. 3-4, pp. 185-396, 2015. 
[50] L. Van der Perre, E. G. Larsson, F. Tufvesson, L. D. Strycker, E. Björnson, and O. Edfors, "Radioweaves for efficient connectivity: analysis and impact of constraints in actual deployments," in Proc. of $53 r d$ Asilomar Conference on Signals, Systems, and Computers, Nov 2019, pp. 15-22. 


\section{Included Papers}





\section{Papers}

The papers associated with this thesis have been removed for copyright reasons. For more details about these see:

https://doi.org/10.3384/9789179292232 


\section{Other Recently Published Theses From \\ The Division of Communication Systems \\ Department of Electrical Engineering (ISY) \\ Linköping University, Sweden}

Unnikrishnan Kunnath Ganesan, Distributed Massive MIMO: Random Access, Extreme Multiplexing and Synchronization, Linköping Studies in Science and Technology. Licentiate Thesis, No. 1923, 2022.

Özgecan Özdogan, Signal Processing Aspects of Massive MIMO and IRS-Aided Communications, Linköping Studies in Science and Technology. Dissertations, No. 2199, 2022.

Amin Ghazanfari, Multi-Cell Massive MIMO: Power Control and Channel Estimation, Linköping Studies in Science and Technology. Dissertations, No. 2142, 2021.

Ziya Gülgün, Physical Layer Security Issues in Massive MIMO and GNSS, Linköping Studies in Science and Technology. Licentiate Thesis, No. 1899, 2021.

Giovanni Interdonato, Cell-Free Massive MIMO: Scalability, Signal Processing and Power Control, Linköping Studies in Science and Technology. Dissertations, No. 2090, 2020.

Ema Becirovic, On Massive MIMO for Massive Machine-Type Communications, Linköping Studies in Science and Technology. Licentiate Thesis, No. 1868, 2020.

Daniel Verenzuela, Exploring Alternative Massive MIMO Designs: Superimposed Pilots and Mixed-ADCs, Linköping Studies in Science and Technology. Dissertations, No. 2041, 2020.

Trịnh Văn Chiến, Spatial Resource Allocation in Massive MIMO Communication: From Cellular to Cell-Free, Linköping Studies in Science and Technology. Dissertations, No. 2036, 2020.

Marcus Karlsson, Blind Massive MIMO Base Stations: Downlink Transmission and Jamming, Linköping Studies in Science and Technology. Dissertations, No. 1950, 2018.

Victor Hei Cheng, Optimizing Massive MIMO: Precoder Design and Power Allocation, Linköping Studies in Science and Technology. Dissertations, No. 1929, 2018.

Christopher Mollén, High-End Performance with Low-End Hardware: Analysis of Massive MIMO Base Station Transceivers, Linköping Studies in Science and Technology. Dissertations, No. 1896, 2017. 


\title{
FACULTY OF SCIENCE AND ENGINEERING
}

\author{
Linköping Studies in Science and Technology, \\ Licentiate Thesis No. 1924, 2022 \\ Division of Communication Systems \\ Department of Electrical Engineering \\ Linköping University \\ SE-581 83 Linköping, Sweden
}

www.liu.se 